\title{
DESIGN AND IMPLEMENTATION OF HIGH SPEED DATA ACQUISITION SYSTEM FOR VIBRATION SIGNAL BASED ON PLC
}

\author{
YANG Dai-hua ${ }^{1}$, LEI Ju-yang ${ }^{2}$ \\ ${ }^{1}$ College of Mechanical Engineering, Shanghai University of Engineering Science, shanghai 201620, China \\ ${ }^{2}$ College of Mechanical Engineering, Shanghai University of Engineering Science, shanghai 201620, China
}

\begin{abstract}
During the actual production process, the upper computer is required to record the process data of high-speed change. For general network communication mode, the maximum refresh rate that WinCC can provide is 250ms, but WinCC can realize highspeed data collection of s7-400plc with the method of RawData archive data link. The acquisition of vibration signal and the acquisition of general analogue signal have some similarities, but there are more differences. For fault diagnosis of vibration signal is the demand of high speed data sampling and WinCC cannot satisfy high refresh rate of rapid real-time data acquisition and display problems, put forward and discussed by using S7-400 PLC in SFB37 system function block (AR_Send) to implement the WinCC the principle and method of high-speed data acquisition. Finally, the simulation results of vibration signal are obtained.
\end{abstract}

Keywords: RawData; High-speed data collection; WinCC; S7-400 PLC

$* * *$

\section{THE INTRODUCTION}

WinCC is a control configuration software in the industrial control system. It is used with the PLC of Siemens S7 series to monitor the production process. For the current network communication mode, the WinCC can provide highest sampling rate is $500 \mathrm{~ms}$, and general field signal, its measurement and control the required data cycle is not high, usually $1 \sim 2$ seconds, sampling period, is enough to meet our the monitoring accuracy of the signal on the spot. However, when the vibration signal is collected and recorded, it is not sufficient. Locomotive vibration diagnosis of rolling bearing, for example, record the signal, can accurately know the cycle of bearing movement is normal, in order to judge of the rolling bearing fault and fault severity. The WinCC configuration cannot meet the requirements of special data collection, so it is necessary to find better ways to realize the purpose of high-speed data collection. Therefore, we try to use WinCC as the upper computer and S7-400plc to solve the problem in the lower computer mode.

\section{PRINCIPLE OF HIGH-SPEED DATA}

\section{COLLECTION}

Conventional WinCC data collection is through the process of its variable address to cycle variables corresponding address data access, and value is a single process, its access cycle $(500 \mathrm{~ms})$ than $(10 \mathrm{~ms})$ PLC scan cycle, so this way of data collection cannot take full advantage of the characteristics of PLC rapid sampling data, and completely determined by the updating cycle of WinCC defined. Acquisition principle is to PLC collected every cycle the value stored in a certain order in the process of a certain format of the DB block, when after reaching a certain number, PLC call system function block SFB37 (AR_Send) will the DB block the initiative to send WinCC, then WinCC automatically in the background call DLL to disassemble the data standardization, and preserved in the WinCC database in chronological order.

In principle analysis, high speed data acquisition and general data acquisition of the main difference is that the former takes the form of packets to send data, the sampling values of a packet contains a n time, completely break through 500 ms WinCC system sampling period, and in the S7-400 the establishment of a data storage, data packets can be before the next packet arrival at any time to transfer, not because the moment different WinCC received packets and produce samples values time mark error, broke through the value system of a single process sampling requirement for real time, achieve the goal of the high speed data acquisition.

\section{DESIGN OF HIGH-SPEED DATA ACQUISITION SYSTEM}

Only S7-400plc has the capability of high-speed data collection, so $\mathrm{S} 7-400$ and WinCC must be selected to perform the configuration to realize the collection function of the field signal and store the data in the upper computer database. PLC will be collected in each cycle the value stored in a certain order in the process of a certain format of the DB in the block, when after reaching a certain number, can call the system function block SFB37 (AR_Send), will the $\mathrm{DB}$ of the initiative to send WinCC, WinCC will then automatically in the background call DLL to disassemble the data standardization, and stored in the database in chronological order. Because it is bulk transmission, can 
effectively improve the communication rate, have made it possible to high-speed data acquisition, and the so-called acquisition frequency will depend on your preserved DB block of the definition, the time interval between the processes of the definition of the minimum time interval can be 1 millisecond.

\subsection{Variable Connection of Configuration Wince}

In order to open the PLC to send packets and displayed in the man-machine interface, WinCC need to make the necessary configuration, the following steps: establishing variable to choose address data type and attributes, establish a file variable - conversion DLL - Set AR_ID and AR ID Subnumber.

Add a new connection to the connection, the communication driver selects the SIMATIC S7-300/400, the interface selects $\mathrm{TCP} / \mathrm{IP}$, and the access point selects the corresponding access point or communication device; Set the corresponding PLC communication parameters in the WinCC RT Prof connection, select send/receive the original data block, and enter the corresponding connection number, as shown in the figure.

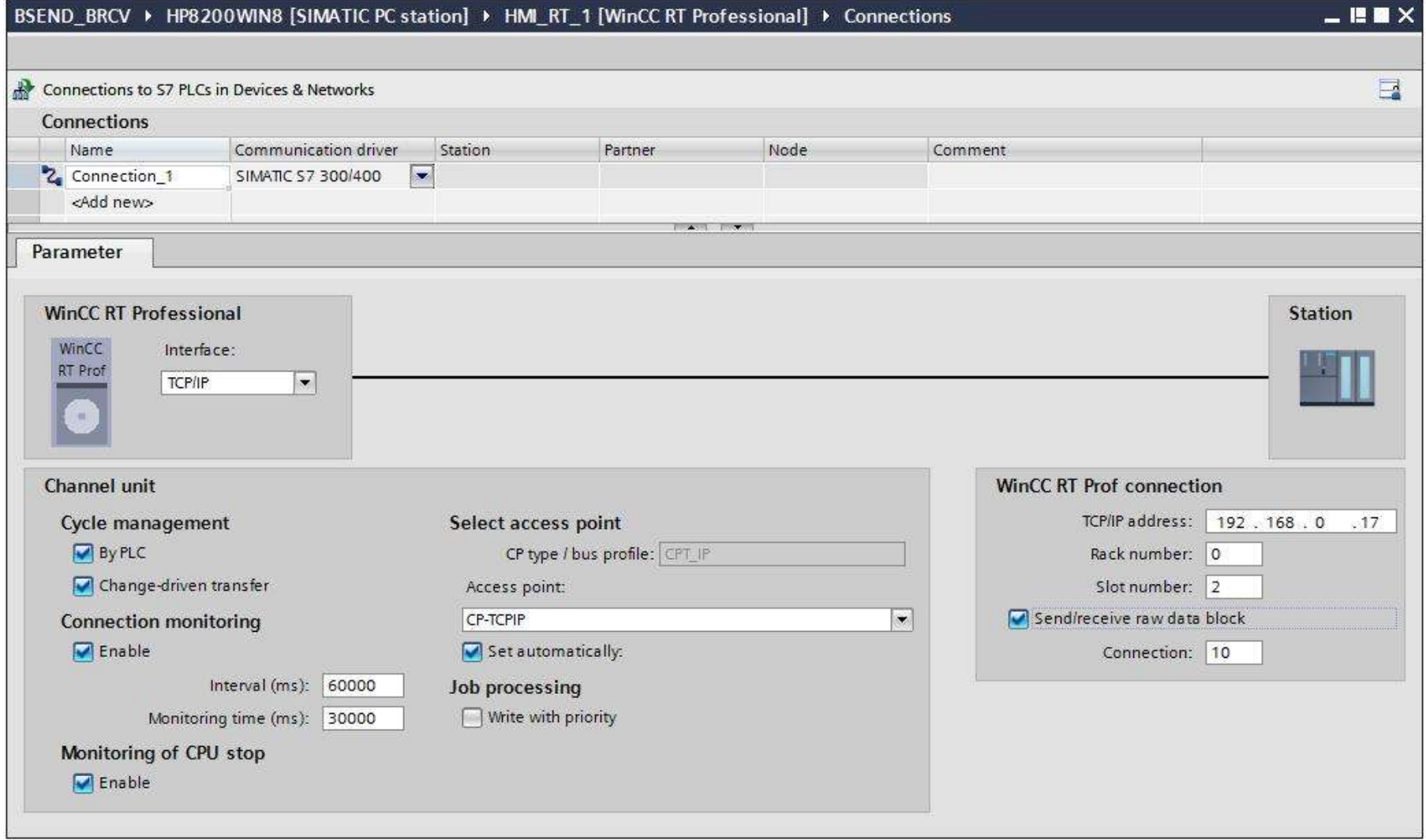

Observation: The connection number should be consistent with the S7 connection number of the configuration in Step7.

\subsection{Parameter Setting}

(1) Insert a DB block in the S7-400 to store the process data and send them to the WinCC database. The block must be defined in a certain format and order, and the key parameters and array of variables are set as follows:

Headertype $1=$ Headertype $2=9$;

NumOfPV1 $=$ NumOfPV2 $=100$;

Cycle $1=$ Cycle $12=10$;

Unit_Type $1=$ Unit_Type $2=1$;

Unit_Range $1=$ Unit_Range $2=3$.

AR_ID_SubNum $1=1$;

AR_ID_SubNum2 $=2$;

Datatype $1=2$ Datatype $2=2$.
(2) Create a new functional block FB1, which defines a static variable, AR_Send, whose type is SFB37; Define an input variable, AR_ID, whose type is DWORD, and then right-click the variable, adding S7_serber and S7_a_type attributes to its object properties; Call AR_Send in FB1 and assign parameters;

(3) Create a new FC1, create two local variables, ReturnValue and Date_And_Time, call FB1 in FC1, and DB11 will be generated automatically.

(4) Define a FC3 to specify the size of the packet to be sent. To enable FC3 to be called at each cold start or hot start, insert OB100 and OB101 to call FC3; Call FC1 in OB. 


\subsection{The Communication Connection between Wincc} and $\mathbf{S 7 - 4 0 0}$

Under the established S7 connection, create a new variable, AR_Send, for the original data type, and the property is set to: archive data connection. Open the WinCC variable record editor and create a new process value archive. Rightclick this archive and select "new process control variables"; Click the "options" button, set AR_Send to 1, AR_Send_Subnumber to 1, click ok, and then create an archive variable. Open the WinCC graphics editor, create a new image, place two IO domains to connect two separate Online tables and select the archived variables. After the configuration is completed, activate the WinCC running system.

\section{WINCC RECEIVING PROCESSING AND SIMULATION RESULTS}

For WinCC receiving treatment, the block of data receiving is mainly around the WinCC archived data link function, the function can will organize a good solid block receiving data, and the standardization of DLL is called to take apart the data, send in the PLC data according to the time sequence of stored in the WinCC database. The work is done in the background. After the data is sent by the PLC, the WinCC will process it automatically. When you need to display, you can insert Online Table into the picture.

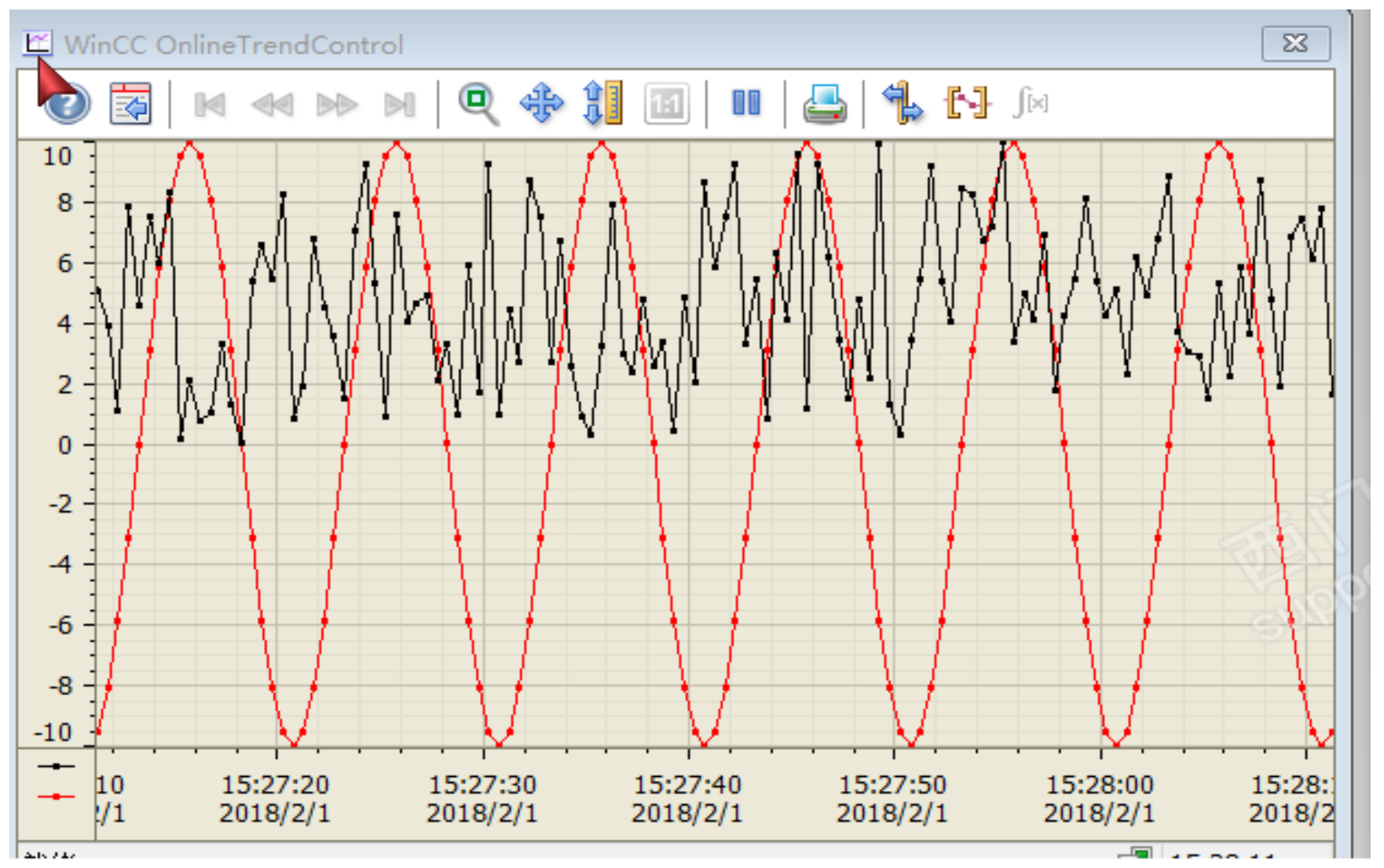

\section{CONCLUSION}

In this paper, the principle and method of WinCC highspeed data acquisition are discussed. With the use of s7-400 PLC and WinCC, we have successfully realized the acquisition of high-speed data of sinusoidal signal and vibration signal, and the collection period reaches $10 \mathrm{~ms}$. It can make full use of the PLC data acquisition of the advantages of quickness, achieve the goal of high speed data, compared with conventional data acquisition methods greatly reflects its superior performance, for the future of vibration signal data acquisition has played a certain role.

\section{Author's Brief Introduction}

YANG Dai-hua, male, han, graduate student of Shanghai university of engineering and technology, research direction: deep learning.

\section{REFERENCES}

[1] Lu Jian-hua. Using WinCC to realize monitoring [M]. Wuhan: journal of wuhan university of science and technology, 2000.

[2] Yang Lu-ming, ray silver. Application of WinCC in automatic monitoring system [J]. Computing technology and automation, 2003,22 (4) 
[3] Wang Shu-guang, Yang Chun-jie. Introduction and development of s7-300/400 [M]. Beijing: People's post and telecommunications press, 2001.

[4] SIMATIC System Software for s7-300/400 System and Standard Functions.

[5] Yu Dan,Yu Jian-shun.STEP simulation debugging and implementation of simulation with WinCC connection $[\mathrm{J}]$. Modern electronics technology, 2010 (14) : 81-83.

[6] Zou Tong. Programming skills of Wincc configuration software $[\mathrm{J}]$. Chemical automation and instrumentation, 2001, (2) : 63-65.

[7] Yang Yuan. Analysis of the design and development techniques of s7-300/400plc system [M]. Machinery industry press, 2008. 